\title{
Measurements with uniform aggregated weighting using boxcar filters for time-synchronised metering, power quality assessment, and control
}

\author{
Andrew J. Roscoe \\ Department of Electronic and Electrical Engineering, \\ University of Strathclyde \\ Glasgow, UK. \\ Andrew.J.Roscoe@strath.ac.uk
}

\begin{abstract}
Boxcar (rectangular) window functions are commonly used for measurements of electrical energy for revenue purposes, or for power quality assessment. This is because they can be repeated using Bartlett's method so that the time-domain sampled data is used with a uniform aggregated weighting. However, such simple window functions are not suitable for real-time protection and control measurements due to their poor frequency-domain performance. Raised-cosine or Tukey windows offer a compromise using Welch's method, but real-time implementation of these functions, using windows adaptive to varying system frequency, can be difficult due to computational load. Alternatively, this paper shows that fast-toexecute cascaded boxcar filters can be used to achieve the desirable property of uniform aggregated weighting, as well as their previously-demonstrated frequency-domain capabilities. This means that measurements based on cascaded boxcar filters could be used for formal revenue metering and power-quality assessment, at the same time as for real-time control, phasor measurement, and protection.
\end{abstract}

Keywords- Power system measurements, Fourier transforms, Frequency measurement, Phase estimation, Power system harmonics, Power system interharmonics, Power conversion harmonics, Industrial power system harmonics, Power system stability, Power system state estimation, Power system parameter estimation.

\section{INTRODUCTION}

When assessing total energy flow or revenue metering across a boundary, one of the major requirements is that the measurement must weight all measured sample values equally. The measurement should not be biased towards certain snapshots in time. If the measurement was biased, then transient or aperiodic events might be over or underrepresented in the final indicated values. The over or underrepresentation could be random, depending on the timealignment (or not) of the events with the higher-weighted or lower-weighted samples. As an example, aperiodic (e.g. interharmonic) effects need to be considered carefully at the $\mathrm{AC}$ and DC terminals of HVDC links which connect AC networks at different $\mathrm{AC}$ frequencies. Harmonics and mixing products of both system $\mathrm{AC}$ frequencies may be present on both $\mathrm{AC}$ systems, and the DC link. Accurate energy measurement is important, since an error of just $0.1 \%$ on an aggregate $1 \mathrm{GW}$ HVDC link over 1 year, at an energy cost of $€ 50 / \mathrm{MWh}$, represents almost $€ 0.5$ million.

\section{A. Windows allowing Uniform Aggregated Weighting (UAW)}

To create an unbiased measurement, a requirement is that repeated measurements using a window function can be aggregated together, in such a way that the aggregated weighting of the input samples is constant (uniform) and unbiased. In this paper, this is referred to as the property of Uniform Aggregated Weighting (UAW). To achieve UAW, there are four possibilities.

The first possibility is to use a boxcar (rectangular) window function, which can be repeated at intervals equal to the boxcar length, revealing UAW across all input samples. This is referred to in literature as Bartlett's method.

The second possibility is that the time-domain window function can "tessellate" exactly with itself and the measurement repetition interval carefully coordinated with the window shape and "tessellation" properties so that the aggregation of the repeated windows leads to UAW. The most obvious examples of this are triangular or raised-cosine (e.g. Hanning or Hamming) windows with length $T$ seconds, repeated at an interval of $T_{r}=T / 2$ seconds. These examples are demonstrated in Fig. 1 and Fig. 2. Another option would be the Tukey window, which consists of a flat central section with raised-cosine ends. The use of repetition intervals less than the window filter length in this way is known as Welch's method.

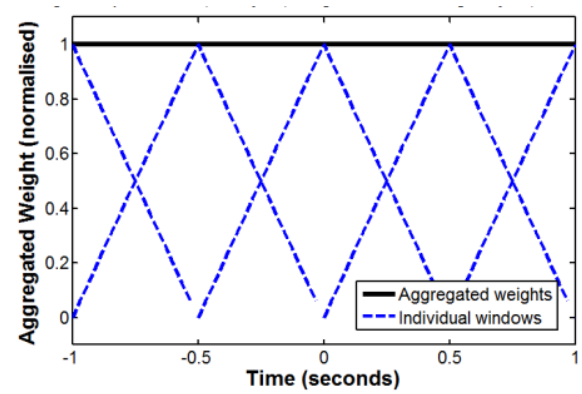

Fig. 1. Triangular window length $T=1 \mathrm{~s}$, repeated with $T_{r}=0.5 \mathrm{~s}$ 


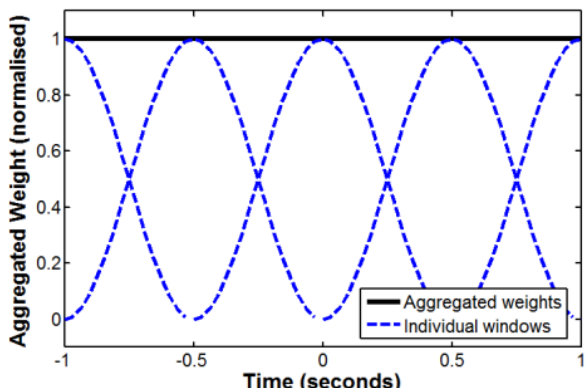

Fig. 2. Hanning window with length $T=1 \mathrm{~s}$, repeated with $T_{r}=0.5 \mathrm{~s}$

The third option is that the window function may not exactly tessellate with itself, but has "other special properties" such that multiple overlaid windows with carefully chosen repetition intervals lead to the property of UAW using Welch's method. Only certain window functions will have these properties with useful measurement repetition intervals. One of these is the raised-cosine window with length $T$ seconds, which can be repeated using Welch's method every $T / M$ seconds, where $M \in \mathbf{N}$ and $\mathrm{M} \geq 2$ to achieve UAW, as will be shown later.

The fourth option is that any window function displays the UAW property, even asymmetric IIR (infinite impulse response) windows, if the repetition interval is reduced towards zero (for continuous functions) or is reduced to 1 sample within discrete digital implementations.

If a window which satisfies options 1-3 is used to provide the UAW property, an additional consideration is the available repetition rates which lead to UAW for that window, and whether these available rates are convenient and useful for the application. For example, within PMU, and emerging "Harmonic PMU" applications, repetition rates at $20 \mathrm{~ms}$ (for $50 \mathrm{~Hz}$ systems) and $16.666 \mathrm{~ms}$ (for $60 \mathrm{~Hz}$ systems) are among the desirable rates. Slower rates might be useful for synchronised power quality assessment.

If the fourth option is used, this may require significant computational power to calculate the windowed transforms at the full sample rate, unless a "Recursive DFT" approach is used, such as in [1]. A problem with relying on this fourth option is that the results must also be communicated to the enduser at the full sample rate, which may exceed communication bandwidth availability. Down-sampled or decimated results will only retain the UAW property if they are integrated/averaged at the full sample rate before being decimated. Note this this is equivalent to adding a single extra cascaded boxcar filter with a time length equal to the downsampled period, and more of this later in section VII!

\section{B. Desirable windows for real-time control and protection}

The frequency-domain performance of a window function also needs to be carefully considered when making measurements within power systems for real-time control and protection purposes. For some functions, such as phasor measurements, the frequency-domain performance requirements are very strict, and this can lead to complex timedomain weighting functions [2] [3]. Such complex windows are highly desirable to produce measurements of voltage, current and power-flow which contain low noise and ripple in the presence of imperfect power quality. If ripple and noise are not filtered out, use of such measurements as inputs to control systems can lead to injection of active or reactive power from primary hardware at the frequencies of the measurement ripple. To reject harmonics, inter-harmonics, flicker and noise requires filters with high broadband rejection outside the passband, and low spectral leakage between harmonics. Filter zeros are often deliberately placed at specific frequencies to reject unwanted signals, leading to specific window functions. However, the passband is required to be relatively flat to provide the greatest calibration accuracy.

Simple uniform-weighting (rectangular) windows are not generally suitable for such measurements, due to their relatively poor characteristics in the frequency domain. So, more complex windows are used. There are many windows to choose from, including: Raised-Cosine (Hanning, Hamming), Tukey, Gaussian, Kaiser, Blackman, Nuttall, BlackmanNuttall, Blackman-Harris, Flat-top, Truncated-sinc ("Brick wall"), Triangular or Parzen. Other windows, and combinations of windows, lead to a myriad of possible window functions [4]. Often, the property of UAW is not considered a priority when choosing a window for a control or protection device.

\section{Conflict between frequency-domain filtering and $U A W$}

Comparing the requirements for energy metering and realtime control can therefore lead to a conflict in choice of measurement window, between one offering UAW (good for unbiased metering) vs. one offering good real-time frequencydomain filtering. However, if a window function can be shown to possess both sets of properties, it would allow both types of measurement to be made on a single instrumentation platform with a minimisation of computational hardware. For example revenue metering, phasor measurement, power flow, and power quality parameters could all be assessed accurately by a single device with excellent time synchronisation.

One example of such a device which attempts to make this crossover is the Arbiter 1133A device. In this device, a fixed Hanning window with $T=100 \mathrm{~ms}$ is used since this offers reasonable performance in the frequency domain, and also the UAW property if repeated using Welch's method as described above, with the repetition interval $T_{r}=T / 2=50 \mathrm{~ms}$ (or any $T_{r}=T / M$, where $M \in \mathbf{N}$ and $\mathrm{M} \geq 2$ ), synchronised accurately to the UTC second rollovers. This time synchronisation of results is a major requirement for a "Harmonic PMU", and allows synchronised power quality assessment and metering with other devices across a network [5]. However, it is a deviation from the power-quality measurement guide IEC 61000-4-30 [6] which recommends a 10-cycle (for $50 \mathrm{~Hz}$ ) or 12-cycle (for $60 \mathrm{~Hz}$ ) boxcar window of adaptive length $10 / f$ or $12 / f$ (normally $\sim 200 \mathrm{~ms}$ ) to suit the actual real-time system frequency $f$. IEC 61000-4-7 [7] allows a Hanning window to be used if the length is not synchronised to the real-time system frequency, but suggests this is mainly a fall-back option. The adaptive filtering specified in [6] \& [7] is useful to minimise spectral leakage within the harmonics. However, using the prescribed rectangular window does not allow UAW to be achieved when frequency is off-nominal, if reports are desired at fixed intervals synchronised to UTC second rollovers (unless results 
are re-calculated every sample by option four above). The simple rectangular window also has very poor frequencydomain properties. Arbiter use a frequency-domain technique to deal with leakage from their fixed-filter Hanning window [5], which works for frequencies close to nominal, making the argument that time-synchronised results possessing the UAW property (and better stop-band rejection) are important enough to justify deviation from [6] \& [7].

Alternatively, the point of this paper is to show that cascading boxcar filters together with themselves, or with other window functions, can be used as a tool to create windows which have the UAW property at user-specified, useful, convenient repetition rates. By understanding the rules of the effects of the boxcar filters on filtering and UAW, FIR filters can be created which both have controllable zero positions for off-nominal frequency operation and minimal spectral leakage, combined with the UAW property at fixed repetition rates synchronised to UTC second rollovers.

\section{THE USE OF CASCADED BOXCAR FILTERS}

Previous work by the author has shown that cascaded boxcar windows can provide a versatile way of achieving good frequency-domain performance, adaptive to varying system frequency $f$, capable of delivering time-synchronised measurements for PMUs compliant with C37.118.1a [3], and with execution times that allow the algorithms to be executed every computational frame at sample rates exceeding $10 \mathrm{kHz}$ [1] [8] [9]. So, while such windows clearly have merit, can they also, in general, be used to achieve the UAW property, to enable them to be used for accurate revenue metering or for formal power quality applications?

A single boxcar window can clearly be used to achieve UAW as previously described. In addition, a triangular window can be considered as the "B-spline" 2-fold convolution of a boxcar window with itself. The symmetry of this window shows that this window also can be used to achieve the UAW property. But what about more complex cascades of multiple boxcar filters with unequal lengths? Does the UAW property break down or does it hold? A theorem is presented here that states:

"For any FIR (Finite Impulse Response) filter which is created by cascading $N$ component boxcar filters in real time, each with time lengths $T_{i}(i=1 . . N)$, the aggregation of successive repetitive measurements using the overlaid filters at regular time intervals of any $T_{r}=T_{i} / M$ results in a uniform aggregated weighting value for all input samples, for any $i=1 . . N$ and any positive integer M."

\section{PROOF}

Proof of the theorem (1) can be demonstrated by taking the Fourier transform of the component boxcar sections, and then multiplying their responses in the frequency domain, which is equivalent to convolution in the time domain. Then, the timeshift translation theorem can be applied, to calculate a summation, in the frequency domain, of the total frequency response of the overall filter, when repeated measurements are made at regular repetition intervals $T_{r}$.

First, for each boxcar component, the well-known Fourier transformation relationship for a boxcar (rectangular) function is used.

$$
f_{\text {boxar }}(t)=\left\{\begin{array}{ll}
0 & \left(|t|>\frac{T}{2}\right) \\
\frac{A}{2} & \left(|t|=\frac{T}{2}\right) \\
A & \left(|t|<\frac{T}{2}\right)
\end{array} \mapsto F_{\text {boxcar }}(f)=A T \frac{\sin (\pi f T)}{(\pi f T)}\right.
$$

Using (2), the Fourier transform of the cascaded boxcar sections can be written in the frequency domain as the product of the response of each boxcar component.

$$
F_{\text {filter }}(f)=\prod_{i=1}^{N}\left(A_{i} T_{i} \frac{\sin \left(\pi f T_{i}\right)}{\left(\pi f T_{i}\right)}\right)
$$

Because the Fourier transformation satisfies the linearity theorem, a summation of terms using the time-shift translation theorem can be applied, which will reveal the frequencydomain response $F_{a g}$ of an infinite series of aggregated measurements made at repeating time intervals given by $T_{r}$.

$$
F_{a g}(f)=\sum_{k=-\infty}^{\infty} e^{-j 2 \pi f k T_{r}}\left(\prod_{i=1}^{N}\left(A_{i} T_{i} \frac{\sin \left(\pi f T_{i}\right)}{\left(\pi f T_{i}\right)}\right)\right)
$$

To prove the theorem, we set $T_{r}=T_{l} / M$ where $(M \in \mathbf{N})$. Note that $T_{r}$ could be set to any $T_{i}$ with the same general result in the following steps. Here it simply makes notation easier if $T_{I}$ is chosen.

$$
F_{a g}(f)=\sum_{k=-\infty}^{\infty} e^{-j 2 \pi f k \frac{T_{1}}{M}}\left(A_{1} T_{1} \frac{\sin \left(\pi f T_{1}\right)}{\left(\pi f T_{1}\right)}\right)\left(\prod_{i=2}^{N}\left(A_{i} T_{i} \frac{\sin \left(\pi f T_{i}\right)}{\left(\pi f T_{i}\right)}\right)\right)
$$

Now the expression can be rearranged slightly, since the righthand terms are independent of $k$.

$$
F_{a g}(f)=\left(\prod_{i=2}^{N}\left(A_{i} T_{i} \frac{\sin \left(\pi f T_{i}\right)}{\left(\pi f T_{i}\right)}\right)\right)\left(A_{1} T_{1} \frac{\sin \left(\pi f T_{1}\right)}{\left(\pi f T_{1}\right)}\right) \sum_{k=-\infty}^{\infty} e^{-j 2 \pi f k \frac{T_{1}}{M}}
$$

It can be observed that (6) has the form:

$$
F_{a g}(f)=A(f) \times B(f) \times C(f)
$$

The proof depends on the shape and features of $B(f)$ (the boxcar filter response of interest) and $C(f)$ (the expression describing the infinite sum of repeated measurements).

It can be seen that:

$$
B(f)=\left(A_{1} T_{1} \frac{\sin \left(\pi f T_{1}\right)}{\left(\pi f T_{1}\right)}\right)
$$


Therefore $B(f)$ has a finite value of $A_{l} T_{l}$ at $f=0$, and values of zero at every $f=1 / T_{l}$. This is the familiar sinc function transformation of a boxcar filter:

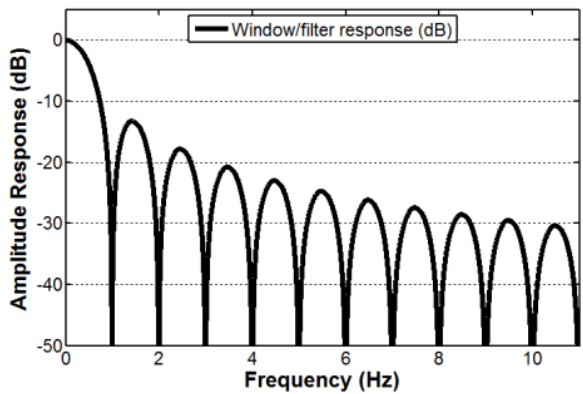

Fig. 3. Response of an example boxcar filter with $A=1$ and $T=1 \mathrm{~s}$

On the other hand:

$C(f)=\sum_{k=-\infty}^{\infty} e^{-j 2 \pi f k \frac{T_{1}}{M}}$

$C(f)$, will become infinite for any $f= \pm M / T_{1_{-}}(M \in \mathbf{N})$, or if $f=0$. But, it will add to zero for any other value of $f$, as the infinite sum of a unity-magnitude rotating phasor. This shows that $C(f)$ can be represented by a regular sequence of delta functions, placed at every value of $f=m T_{l}$, where $\mathrm{m}$ is any integer $(m \in \mathbf{Z})$.

$C(f)=\frac{M}{T_{1}} \delta\left(f-m T_{1}\right) \quad(m \in \mathbf{Z})$

Comparing the features of $B(f)$ and $C(f)$, it can be seen that $B(f)$ contains zeros which exactly coincide with every deltafunction in $C(f)$, except the delta-function which occurs at $f=0$. This simply reveals the combination of $B(f)$ times $C(f)$ to be:

$B(f) \times C(f)=A_{1} T_{1} \frac{M}{T_{1}} \delta(f)$

This result can be substituted back into (7) and (6) to give:

$$
\begin{aligned}
& F_{a g}(f)=\left(\prod_{i=2}^{N}\left(A_{i} T_{i} \frac{\sin \left(\pi f T_{i}\right)}{\left(\pi f T_{i}\right)}\right)\right)_{f=0} A_{1} T_{1} \frac{M}{T_{1}} \delta(f) \\
& F_{a g}(f)=\left(\prod_{i=2}^{N}\left(A_{i} T_{i}\right)\right) A_{1} T_{1} \frac{M}{T_{1}} \delta(f) \\
& F_{a g}(f)=\left(\prod_{i=1}^{N}\left(A_{i} T_{i}\right)\right) \frac{\delta(f)}{T_{r}} \quad\left(T_{r}=\frac{M}{T_{1}}\right)
\end{aligned}
$$

Finally, inverse Fourier-transforming (14) using the standard relationship for a delta-function, reveals that in the time domain, the aggregate weightings of the overlaid repeated measurements can be described by a constant value, i.e. a uniformly weighted sample set.

$$
f(t)=\frac{\prod_{i=1}^{N}\left(A_{i} T_{i}\right)}{T_{r}}
$$

(independent of $t$, so constant and uniform for all $t$ )

Often the amplitude of each boxcar filter weighting will be set to $A_{i}=1 / T_{i}$, so that each boxcar filter (and the cascaded combination) provides an averaged value. In this case:

$$
f(t)=\frac{1}{T_{r}}
$$

(independent of $t$, so constant and uniform for all $t$ )

\section{GENERAL RULES FOR ALL WINDOW TYPES}

In general, by the same argument given by (7) et. seq., to achieve the property of UAW, the frequency-domain performance of any window must contain zeros at every integer multiple $N$ of the repetition frequency $1 / T_{r}$ at which the results are aggregated.

"To achieve uniform aggregated weighting with a window function at a repetition interval $T_{r}$, its frequency-domain transform must have zeros placed at every frequency $N / T_{r}(N \in \mathbf{N})$ "

\section{IMPLICATIONS FOR NON-BOXCAR WINDOW TYPES}

Using this knowledge, it is easy to now confirm that, for example, the Hanning window of length $T$ can be repeated at any interval $T / M$ seconds, where $M \in \mathbf{N}$ and $\mathrm{M} \geq 2$, because it has zeros at every $N M / T \mathrm{~Hz}$ where $N \in \mathbf{N}$ (Fig. 4).

A carefully-designed Flat-top window can also achieve the UAW property if repeated at any interval $T_{r}=T / M$ where $M \in \mathbf{N}$ and $\mathbf{M} \geq 5$. This is not intuitive whatsoever from its time-domain shape (Fig. 5) which even includes negative weights, but can be easily understood by reference to its frequency response which has regular zeros at $N M / \mathrm{T} \mathrm{Hz}$ where $M \geq 5$ (Fig. 6).

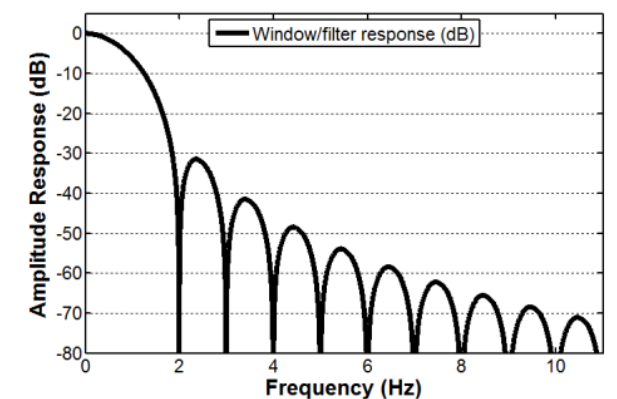

Fig. 4. Hanning window (length $T=1 \mathrm{~s}$ ) response: zeros at every multiple of $M / T \mathrm{~Hz}$ where $M \in \mathbf{N}$ and $\mathrm{M} \geq 2$.

This is a postprint of a paper submitted to and presented at the IEEE International Instrumentation and Measurement Technology Conference (I²MTC), 2015 [http://dx.doi.org/10.1109/I2MTC.2015.7151377], and is subject to IEEE copyright. 


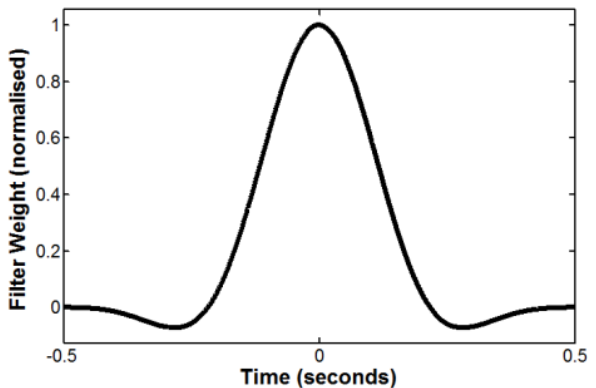

Fig. 5. Flat-top window (length $T=1 \mathrm{~s}$ )

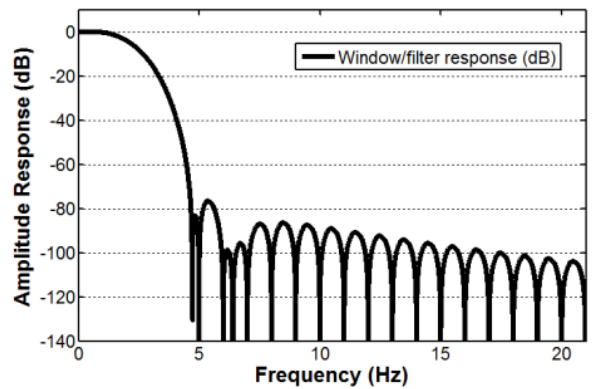

Fig. 6. Flat-top ( $T=1 \mathrm{~s}$ ) response: zeros at every $M / T \mathrm{~Hz}$ where $\mathrm{M} \geq 5$

For other window functions, care is required and each window must be carefully examined and assessed. For example, a Gaussian window with $\sigma=0.25$ has a response as shown in Fig. 7. It appears to have regular zeros but they are placed at non-intuitive locations and may not be exactly equally spaced. Setting $T_{r}=1 / 6.1676$ for $T=1 \mathrm{~s}$, by zooming in on Fig. 7 to locate the zero as accurately as possible, results in close-to UAW, but not exactly. This repetition rate is not particularly convenient. A full analysis of all window types is beyond the scope of this paper.

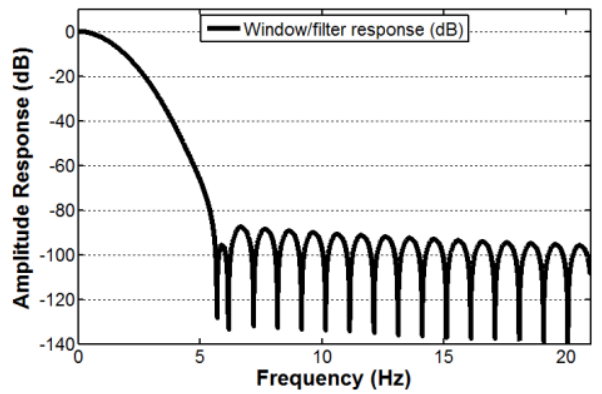

Fig. 7. Gaussian window (length $T=1 \mathrm{~s}$ ) response: zeros inconveniently placed.

\section{EXAMPLES OF CASCADED BOXCARS SHOWING UAW}

By the theorem (1) it has been shown that any overall filter made up of cascaded boxcar sections can be used to obtain the UAW property, and with a range of useful repetition rates to choose from. The available rates can be modified by the user as required, by adjustment of the boxcar sections. In particular the longest available repetition time (lowest rate) is governed directly by the length of the longest boxcar section by (1).

To show simple examples of this, first the Parzen window is presented. The Parzen window is a 4-fold convolution of a boxcar filter and so in Fig. 8 a total of 4 boxcar sections, each with length $0.25 \mathrm{~s}$, are cascaded together to form a single filter of length 1s. By (1) this should exhibit UAW with a measurement repetition interval of $T_{r}=0.25 \mathrm{~s}$, and Fig. 8 shows this to be the case. Zooming in on Fig. 8 shows the Aggregated weights to be exactly unity.

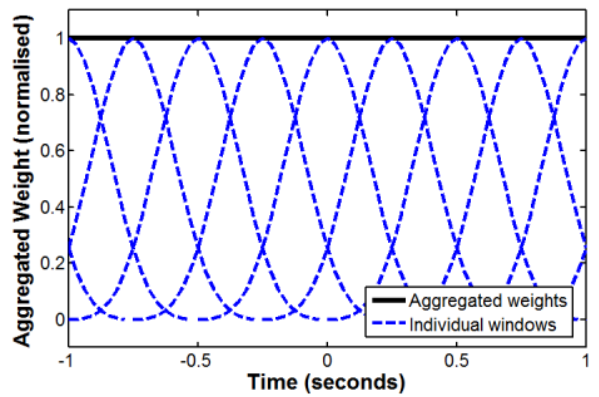

Fig. 8. Parzen window with length $T=1 \mathrm{~s}$, repeated with $T_{r}=0.25 \mathrm{~s}$

The final example is a more complex filter of total length $T=1 \mathrm{~s}$, consisting of three unequal-length boxcar sections: $T_{I}=0.5 \mathrm{~s}, T_{2}=0.3 \mathrm{~s}$, and $T_{3}=0.2 \mathrm{~s}$. The desired property of UAW can be achieved by selecting any of the potentially-convenient rates of:

- $T_{r}=T_{1}=0.5 \mathrm{~s}($ Fig. 9)

- $T_{r}=T_{2}=0.3 \mathrm{~s}$ (Fig. 10)

- $\quad$ or $T_{r}=T_{3}=0.2 \mathrm{~s}$ (Fig. 11)

- or any of the available integer "sub-multiples" of the above by (1).

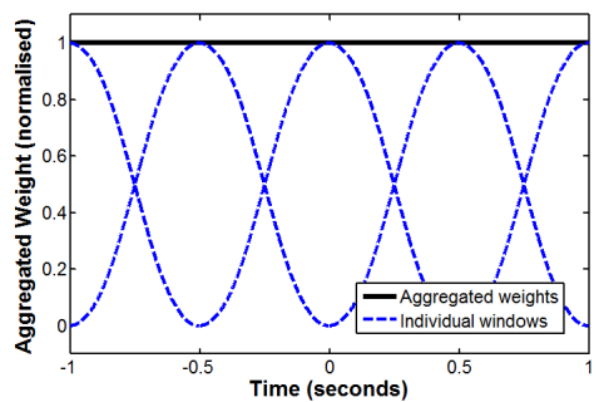

Fig. 9. Cascaded boxcars with lengths $0.5 \mathrm{~s}, 0.3 \mathrm{~s}, 0.2 \mathrm{~s}$ (total length $T=1 \mathrm{~s}$ ), repeated with $T_{r}=0.5 \mathrm{~s}$

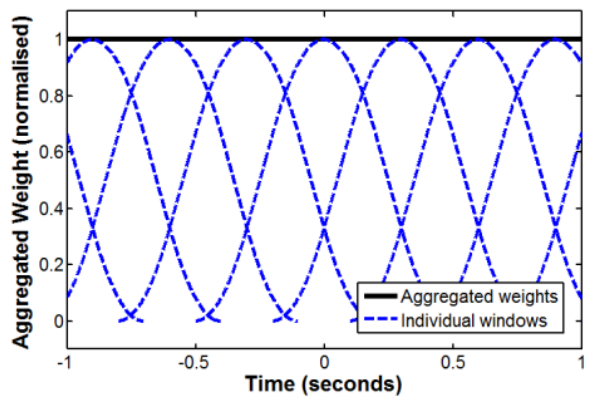

Fig. 10. Cascaded boxcars with lengths $0.5 \mathrm{~s}, 0.3 \mathrm{~s}, 0.2 \mathrm{~s}$ (total length $T=1 \mathrm{~s}$ ), repeated with $T_{r}=0.3 \mathrm{~s}$

This is a postprint of a paper submitted to and presented at the IEEE International Instrumentation and Measurement Technology Conference (I²MTC), 2015 [http://dx.doi.org/10.1109/I2MTC.2015.7151377], and is subject to IEEE copyright. 


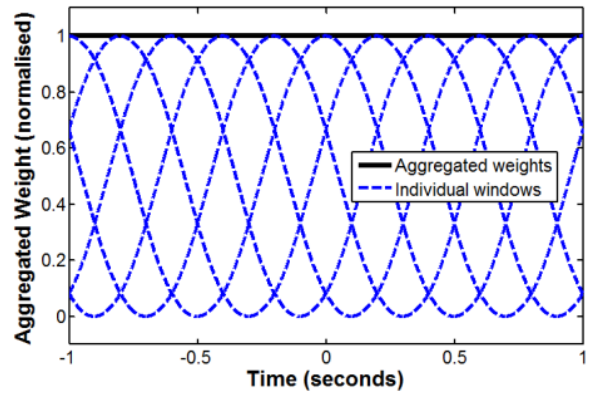

Fig. 11. Cascaded boxcars with lengths $0.5 \mathrm{~s}, 0.3 \mathrm{~s}, 0.2 \mathrm{~s}$ (total length $T=1 \mathrm{~s}$ ), repeated with $T_{r}=0.2 \mathrm{~s}$

\section{CASCADING A SINGLE FIXED-LENGTH BOXCAR TO ANY OTHER WINDOW}

A final observation and third useful rule can also be deduced and stated. By the theorem/rule (17), and by observation of the properties of a single boxcar filter (e.g. Fig. 3 ), it can be seen that cascading (convoluting in the time domain) a single fixed-length boxcar filter of length $T_{r}$ with any other pre-existing FIR filter window function will add the required zeros in the frequency domain to give the UAW property at a repetition interval $T_{r}$, since the resulting frequency domain performance will be the product of the pre-existing filter response and the boxcar response.

"Cascading (convoluting) a single boxcar filter of length $N T_{r}(N \in \mathbf{N})$ with any fixed pre-existing FIR filter window will guarantee the uniform aggregated weighting property with a repetition interval $T_{r}$ ",

This is extremely powerful. It means that, for example, a Gaussian window could be given the UAW property at a more convenient repetition rate by cascading it with a boxcar filter of length $20 \mathrm{~ms}$ (for a $50 \mathrm{~Hz}$ system). Also, it offers the possibility of creating long adaptive filters based on Hanning or boxcar filters with lengths that are multiples of the actual off-nominal system frequency $f$, but then cascading a single extra boxcar filter at a fixed (non-adaptive) length to suit the desired repetition (reporting) rate. This would achieve all three desirable properties:

1. Uniform Aggregated Weighting (UAW).

2. At a convenient and controllable repetition (reporting) rate that can be synchronized with UTC second rollovers.

3. Minimization of spectral leakage through adaptive filters.

The only caveat is the word "fixed" in (18). If the "preexisting" filter is changing in real-time, for instance in response to constantly changing system frequency $f$, then (18) is theoretically violated. An interesting future piece of work is to examine the magnitude of the excursions from UAW due to gradually changing $f$ which cause small step-wise changes in adaptive filter design from window to window. If the magnitude of the UAW excursions can be quantified and guaranteed to be below various thresholds, this might allow fully-adaptive algorithms to operate and satisfy all three conditions above with high and quantifiable accuracy.

\section{VIII.CONCLUSIONS}

To achieve Uniform Aggregated Weighting with a window function at a repetition interval $T_{r}$, its frequency-domain transform must have zeros placed at every frequency $N / T_{r}$. Window functions can be made to provide the property of Uniform Aggregated Weighting at a chosen repetition rate using Welch's method by the cascading of just a single simple boxcar filter with the main filter, which may be a complex adaptive device. If the entire filter is built from cascaded boxcar sections, then Uniform Aggregated Weighting is available at many repetition rates which are easily predictable from the boxcar filter lengths.

Knowledge and use of these rules allows window functions to be created which produce unbiased measurements at useful repetition (reporting) rates that can be synchronized with UTC second rollovers, and that minimize spectral leakage. This allows the same measurement device with the same measurement window and computations, to produce results which are suitable for time-synchronised revenue metering and power-quality functions, and also for real-time control and protection functions. This offers the potential to reduce the cost of instrumentation, by fulfilling multiple functions within a single measurement device, on a single processor unit. Further work is required to fully quantify the effect of dynamicallychanging filter windows on the uniformity of the aggregated weightings during rate-of-change-of-frequency (ROCOF) events.

\section{REFERENCES}

[1] A. J. Roscoe, I. F. Abdulhadi, and G. M. Burt, "P and M Class Phasor Measurement Unit Algorithms using Adaptive Cascaded Filters," IEEE Transactions on Power Delivery, vol. 28, pp. 1447-1459, 2013.

[2] IEEE, "IEEE Standard for Synchrophasor Measurements for Power Systems," C37.118.1-2011, 2011.

[3] IEEE, "IEEE Standard for Synchrophasor Measurements for Power Systems -- Amendment 1: Modification of Selected Performance Requirements " C37.118.1a-2014, 2014.

[4] S. W. Smith, "The Scientist and Engineer's Guide to Digital Signal Processing," ISBN 0-9660176-3-3, 1997. Available: http://www.dspguide.com/, accessed Sept 2014.

[5] B. Dickerson, "Applications of Synchronised Power Quality Measurements," Arbiter Systems, 2014. Available: http://www.arbiter.com/files/productattachments/PD0052000_Applications_Of_Synchronised_Power_Qualit y_Measurements.pdf, accessed Sep $20 \overline{14}$.

[6] IEC, "Electromagnetic compatibility (EMC) - Part 4-30: Testing and measurement techniques - Power quality measurement methods," IEC61000-4-30, 2003.

[7] IEC, "Electromagnetic compatibility (EMC) - Part 4-7: Testing and measurement techniques - General guide on harmonics and interharmonics measurements and instrumentation, for power supply systems and equipment connected thereto," IEC-61000-4-7, 2002-2011.

[8] A. J. Roscoe, "Exploring the relative performance of frequency-tracking and fixed-filter Phasor Measurement Unit algorithms under C37.118 test procedures, the effects of interharmonics, and initial attempts at merging P class response with M class filtering," IEEE Transactions on Instrumentation and Measurement, vol. 62, pp. 2140-2153, 2013.

[9] A. J. Roscoe, "Frequency tracking, C37.118.1 a compliant, P and M class PMU (Phasor Measurement Unit) algorithm examples for MATLAB Simulink," 2014 Available: http://strathprints.strath.ac.uk/48245/, accessed Oct 2014. 
This is a postprint of a paper submitted to and presented at the IEEE International Instrumentation and Measurement Technology Conference (I²MTC), 2015 [http://dx.doi.org/10.1109/I2MTC.2015.7151377], and is subject to IEEE copyright. 